Acta Crystallographica Section E

Structure Reports

Online

ISSN 1600-5368

\section{(E)-6,8-Dichloro-3-\{[(naphthalen-1-yl- methyl)iminiumyl]methyl\}-2H-chromen- 4-olate}

\author{
Yoshinobu Ishikawa* and Yuya Motohashi \\ School of Pharmaceutical Sciences, University of Shizuoka, 52-1 Yada, Suruga-ku, \\ Shizuoka 422-8526, Japan \\ Correspondence e-mail: ishi206@u-shizuoka-ken.ac.jp
}

Received 20 June 2013; accepted 1 July 2013

Key indicators: single-crystal X-ray study; $T=100 \mathrm{~K}$; mean $\sigma(\mathrm{C}-\mathrm{C})=0.002 \AA$; $R$ factor $=0.033 ; w R$ factor $=0.083 ;$ data-to-parameter ratio $=16.5$.

In the title compound, $\mathrm{C}_{21} \mathrm{H}_{15} \mathrm{Cl}_{2} \mathrm{NO}_{2}$, the $\mathrm{H}$ atom of the $-\mathrm{OH}$ group is transferred to the $\mathrm{N}$ atom of the imine, forming a zwitterion. Thus, there is formation of a six-membered ring via an intramolecular $\mathrm{O} \cdots \mathrm{H}-\mathrm{N}$, rather than $\mathrm{O}-\mathrm{H} \cdots \mathrm{N}$, hydrogen bond in the molecule. The dihedral angle between the naphthalene ring system and the benzene ring of the $2 \mathrm{H}$ chromen system is $87.41(4)^{\circ}$. In the crystal, the molecules are packed through $\mathrm{N}-\mathrm{H} \cdots \mathrm{O}, \pi-\pi$ [centroid-centroid distances $=3.744(3)$ and $3.780(3) \AA], \mathrm{C}-\mathrm{Cl} \cdots \pi[\mathrm{Cl} \cdots$ centroid $=$ $3.261(3) \AA], \mathrm{C}-\mathrm{H} \cdots \pi$ and $\mathrm{C}-\mathrm{H} \cdots \mathrm{O}$ interactions.

\section{Related literature}

For the biological propertries of similar structures, see: Khan et al. (2009); Tu et al. (2013). For related structures, see: Benaouida et al. (2013); Małecka \& Budzisz (2006).<smiles>[O-]C1=C(C=NCc2cccc3ccccc23)COc2c(Cl)cc(Cl)cc21</smiles>

\section{Experimental}

Crystal data

$\mathrm{C}_{21} \mathrm{H}_{15} \mathrm{Cl}_{2} \mathrm{NO}_{2}$ $M_{r}=384.26$

$$
\begin{aligned}
& b=8.910(6) \AA \\
& c=12.008(9) \AA \\
& \beta=102.65(4)^{\circ} \\
& V=1700.2(19) \AA^{3} \\
& Z=4
\end{aligned}
$$

Data collection

Rigaku AFC7R diffractometer 4657 measured reflections

3875 independent reflections

3367 reflections with $F^{2}>2.0 \sigma\left(F^{2}\right)$

$$
\begin{aligned}
& \text { Mo } K \alpha \text { radiation } \\
& \mu=0.40 \mathrm{~mm}^{-1} \\
& T=100 \mathrm{~K} \\
& 0.37 \times 0.37 \times 0.28 \mathrm{~mm}
\end{aligned}
$$

\section{Refinement}

$R\left[F^{2}>2 \sigma\left(F^{2}\right)\right]=0.033$

$w R\left(F^{2}\right)=0.083$

$S=1.04$

3875 reflections

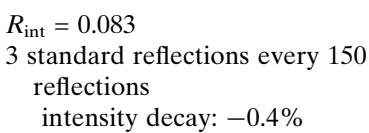

Table 1

Hydrogen-bond geometry $\left(\AA,{ }^{\circ}\right)$.

$\mathrm{Cg} 2$ is the centroid of the $\mathrm{C} 4-\mathrm{C} 9$ ring.

\begin{tabular}{llllc}
\hline$D-\mathrm{H} \cdots A$ & $D-\mathrm{H}$ & $\mathrm{H} \cdots A$ & $D \cdots A$ & $D-\mathrm{H} \cdots A$ \\
\hline $\mathrm{N} 1-\mathrm{H} 6 \cdots \mathrm{O} 2$ & 0.88 & 2.18 & $2.794(2)$ & 126 \\
$\mathrm{~N} 1-\mathrm{H} 6 \cdots \mathrm{O} 2^{\mathrm{i}}$ & 0.88 & 2.54 & $3.306(3)$ & 146 \\
$\mathrm{C} 1-\mathrm{H} 2 A \cdots \mathrm{O} 2^{\mathrm{ii}}$ & 0.99 & 2.52 & $3.472(3)$ & 160 \\
$\mathrm{C} 15-\mathrm{H} 11 \cdots C g 2^{\mathrm{iii}}$ & 0.95 & 2.77 & $3.682(3)$ & 160 \\
\hline Symmetry codes: & $(\mathrm{i})$ & $-x+1,-y+1,-z+1 ;$ & (ii) & $-x+1, y+\frac{1}{2},-z+\frac{3}{2} ; \quad$ (iii) \\
$-x+1,-y+2,-z+1$. & & & &
\end{tabular}

Data collection: WinAFC (Rigaku, 1999); cell refinement: WinAFC; data reduction: WinAFC; program(s) used to solve structure: SHELXS97 (Sheldrick, 2008); program(s) used to refine structure: SHELXL97 (Sheldrick, 2008); molecular graphics: CrystalStructure (Rigaku, 2010); software used to prepare material for publication: CrystalStructure.

We acknowledge the University of Shizuoka for supporting this study.

Supplementary data and figures for this paper are available from the IUCr electronic archives (Reference: ZP2006).

\section{References}

Benaouida, M. A., Chetioui, S. \& Bouaoud, S. E. (2013). Acta Cryst. E69, o867-o868.

Khan, K. M., Ambreen, N., Hussain, S., Perveen, S. \& Choudhary, M. I. (2009). Bioorg. Med. Chem. 17, 2983-2988.

Małecka, M. \& Budzisz, E. (2006). Acta Cryst. E62, o5058-05060.

Rigaku (1999). WinAFC Diffractometer Control Software. Rigaku Corporation, Tokyo, Japan.

Rigaku (2010). CrystalStructure. Rigaku Corporation, Tokyo, Japan.

Sheldrick, G. M. (2008). Acta Cryst. A64, 112-122.

Tu, Q. D., Li, D., Sun, Y., Han, X. Y., Yi, F., Sha, Y., Ren, Y. L., Ding, M. W., Feng, L. L. \& Wan, J. (2013). Bioorg. Med. Chem. 21, 2826-2831. 


\section{supporting information}

Acta Cryst. (2013). E69, o1226 [doi:10.1107/S1600536813018084]

\section{(E)-6,8-Dichloro-3-\{[(naphthalen-1-ylmethyl)iminiumyl]methyl\}-2H-chromen-4- olate}

\section{Yoshinobu Ishikawa and Yuya Motohashi}

\section{S1. Comment}

Schiff bases of 3-formyl chromones have attracted much attention due to their biological functions such as enzyme inhibition (Khan et al. 2009; Tu et al. 2013). Here we report the crystal structure of the title compound, which was obtained from the condensation reaction of 6,8-dichloro-3-formylchromone with 1-naphthylmethylamine and successive reduction with 2-picoline borane. The structure shows that the $\mathrm{H}$ atom of the $-\mathrm{OH}$ group is transferred to the $\mathrm{N} 1$ atom of the imine, thus forming a zwitterion. As a result, an intramolecular $\mathrm{O} \cdots \mathrm{H}-\mathrm{N}[\mathrm{O} 2 \cdots \mathrm{N} 1=2.795(2) \AA]$, rather than $\mathrm{O}-\mathrm{H} \cdots \mathrm{N}$, hydrogen bond is formed. The bond distances O2-C3 [1.245 (3) $\AA], \mathrm{C} 3-\mathrm{C} 2[1.431$ (3) $\AA], \mathrm{C} 2-\mathrm{C} 10[1.377$ (3) $\AA]$ and $\mathrm{C} 10-\mathrm{N} 1[1.329(3) \AA]$ and torsion angles O2-C3-C2-C10 [3.2 (3) $\left.{ }^{\circ}\right]$ and $\mathrm{C} 3-\mathrm{C} 2-\mathrm{C} 10-\mathrm{N} 1\left[-2.4(3)^{\circ}\right]$ in the sixmembered ring indicate charge delocalization among the atoms. This effect might be responsible for the preferential reduction of the $\alpha, \beta$-unsaturated carbonyl of the synthetic intermediate, rather than reduction of the imine. The dihedral angle between the naphthalene ring and the benzene part of the $2 H$-chromen ring is $87.41(4)^{\circ}$. In the crystal, the molecules are packed through intermolecular $\mathrm{N}-\mathrm{H} \cdots \mathrm{O}$, as shown in Figure $2, \pi \cdots \pi, \mathrm{C}-\mathrm{Cl} \cdots \pi, \mathrm{C}-\mathrm{H} \cdots \pi$ and $\mathrm{C}-\mathrm{H} \cdots \mathrm{O}$ interactions.

\section{S2. Experimental}

1-Naphthylmethylamine (5.0 mmol), 6,8-dichloro-3-formylchromone (5.0 mmol) and 2-picoline borane $(5.0 \mathrm{mmol})$ were dissolved in a mixture of $\mathrm{MeOH}-\mathrm{AcOH}(10: 1,60 \mathrm{ml})$, and stirred overnight at room temperature. Hydrochloric acid (1 $\mathrm{M}, 20 \mathrm{ml}$ ) was added to the reaction mixture, which was then stirred for $30 \mathrm{~min}$. After neutralization with saturated $\mathrm{NaHCO}_{3}$, the mixture was extracted with methylene chloride. The extract was washed with brine, dried over anhydrous $\mathrm{Na}_{2} \mathrm{SO}_{4}$ and purified by column chromatography on silica gel ( $n$-hexane: ethyl acetate $\left.=9: 1\right)$. The eluted fractions were concentrated and filtered off. Layering $n$-hexane on the filtrate gave single crystals suitable for X-ray diffraction (yield $19 \%)$.

\section{S3. Refinement}

The carbon-bound hydrogen atoms were placed in geometrical positions [C-H 0.95 to $\left.0.99 \AA, U_{\text {iso }}(\mathrm{H})=1.2 U_{\text {eq }}(\mathrm{C})\right]$, and refined using a riding model. The hydrogen atom of the $\mathrm{OH}$ group was located near $\mathrm{N} 1$ of the imine in a difference Fourier map, and refined with distance constraint $\left[\mathrm{N}-\mathrm{H} 0.88 \AA, U_{\text {iso }}(\mathrm{H})=1.2 U_{\text {eq }}(\mathrm{N})\right]$. 


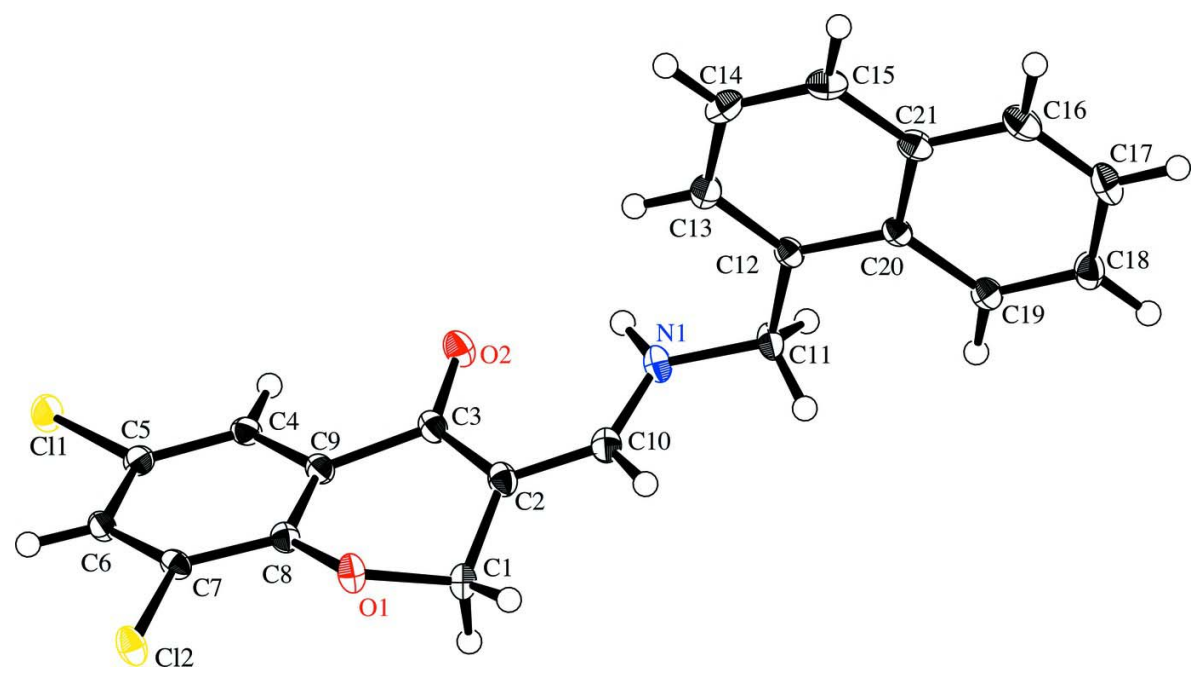

\section{Figure 1}

The molecular structure of the title compound, with displacement ellipsoids drawn at the $50 \%$ probability level. Hydrogen atoms are shown as small spheres of arbitrary radius.

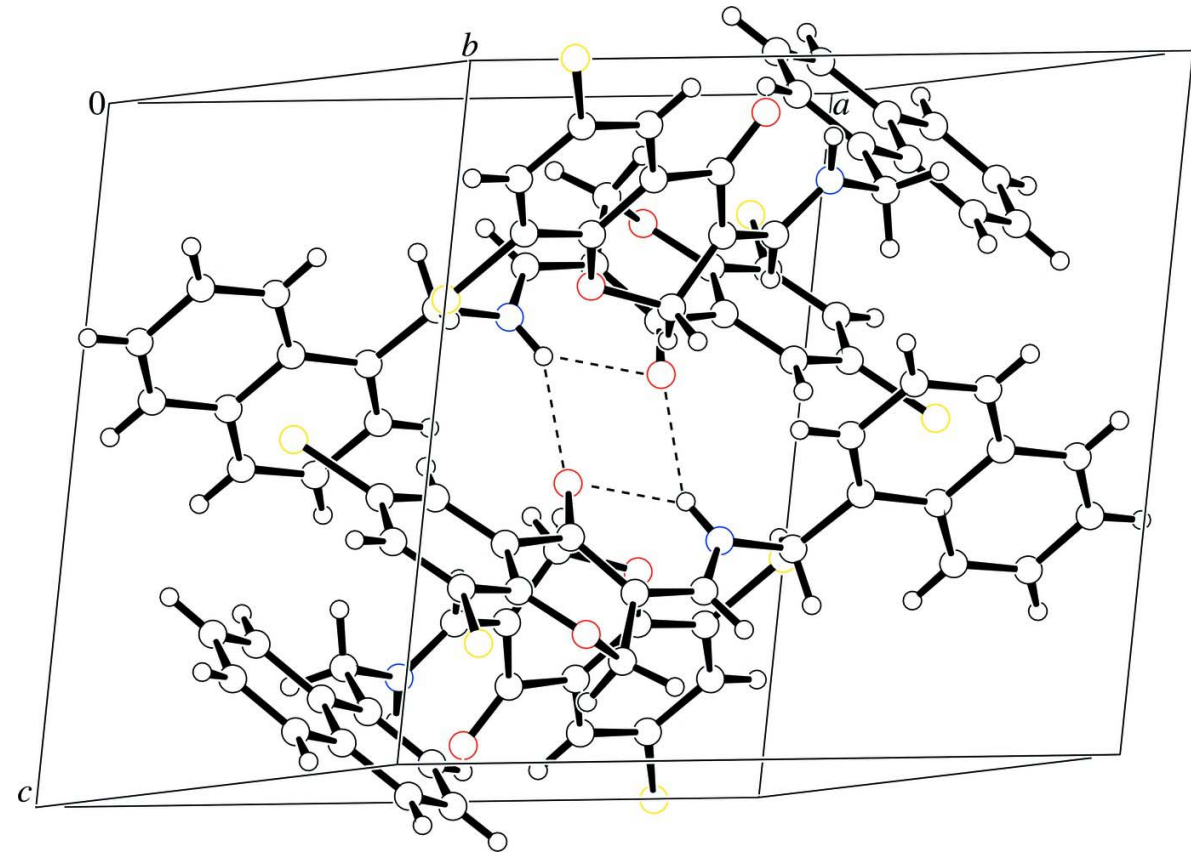

\section{Figure 2}

A crystal packing view of the title compound. Intra- and intermolecular $\mathrm{N}-\mathrm{H} \cdots \mathrm{O}$ hydrogen bonds are represented as dashed lines.

\section{(E)-6,8-Dichloro-3-\{[(naphthalen-1-ylmethyl)iminio]methyl\}-2H-chromen-4-olate}

\section{Crystal data}

$\mathrm{C}_{21} \mathrm{H}_{15} \mathrm{Cl}_{2} \mathrm{NO}_{2}$

$M_{r}=384.26$

Monoclinic, $P 2_{1} / c$

Hall symbol: -P 2ybc

$$
\begin{aligned}
& a=16.286(7) \AA \\
& b=8.910(6) \AA \\
& c=12.008(9) \AA \\
& \beta=102.65(4)^{\circ}
\end{aligned}
$$




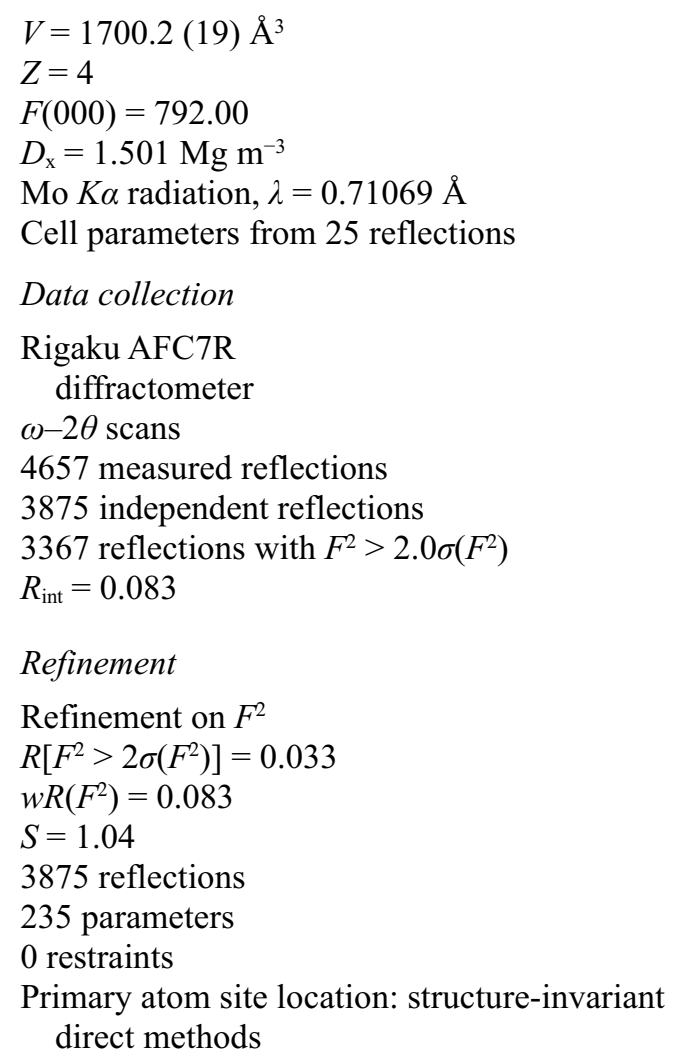

Special details

Refinement. Refinement was performed using all reflections. The weighted $R$-factor $(w R)$ and goodness of fit $(S)$ are based on $F^{2}$. R-factor (gt) are based on $F$. The threshold expression of $F^{2}>2.0 \sigma\left(F^{2}\right)$ is used only for calculating $R$-factor (gt).

Fractional atomic coordinates and isotropic or equivalent isotropic displacement parameters $\left(\AA^{2}\right)$

\begin{tabular}{lllll}
\hline & $x$ & $y$ & $z$ & $U_{\text {iso }} * / U_{\text {eq }}$ \\
\hline C11 & $0.10099(2)$ & $0.41074(4)$ & $0.50407(3)$ & $0.02134(10)$ \\
C12 & $0.17147(2)$ & $0.84582(4)$ & $0.82092(3)$ & $0.01871(10)$ \\
O1 & $0.34501(6)$ & $0.79385(11)$ & $0.80754(9)$ & $0.0176(2)$ \\
O2 & $0.43015(6)$ & $0.52671(12)$ & $0.57813(9)$ & $0.0194(2)$ \\
N1 & $0.58885(7)$ & $0.64807(13)$ & $0.66903(11)$ & $0.0178(3)$ \\
C1 & $0.42933(8)$ & $0.73258(16)$ & $0.84317(12)$ & $0.0173(3)$ \\
C2 & $0.46328(8)$ & $0.67928(15)$ & $0.74377(12)$ & $0.0163(3)$ \\
C3 & $0.40821(8)$ & $0.59345(15)$ & $0.65803(12)$ & $0.0153(3)$ \\
C4 & $0.25901(8)$ & $0.50521(16)$ & $0.59487(11)$ & $0.0149(3)$ \\
C5 & $0.17435(8)$ & $0.52122(16)$ & $0.59411(11)$ & $0.0156(3)$ \\
C6 & $0.14631(8)$ & $0.62656(15)$ & $0.66285(11)$ & $0.0152(3)$ \\
C7 & $0.20515(8)$ & $0.71380(15)$ & $0.73498(11)$ & $0.0141(3)$ \\
C8 & $0.29135(8)$ & $0.69931(15)$ & $0.73910(11)$ & $0.0142(3)$ \\
C9 & $0.31801(8)$ & $0.59510(15)$ & $0.66686(11)$ & $0.0148(3)$ \\
C10 & $0.54680(8)$ & $0.70260(15)$ & $0.74329(12)$ & $0.0166(3)$ \\
C11 & $0.67892(8)$ & $0.67249(16)$ & $0.68340(12)$ & $0.0174(3)$
\end{tabular}

$$
\begin{aligned}
& \theta=15.7-17.5^{\circ} \\
& \mu=0.40 \mathrm{~mm}^{-1} \\
& T=100 \mathrm{~K} \\
& \text { Block, yellow } \\
& 0.37 \times 0.37 \times 0.28 \mathrm{~mm}
\end{aligned}
$$

$$
\begin{aligned}
& \theta_{\max }=27.6^{\circ} \\
& h=-20 \rightarrow 21 \\
& k=0 \rightarrow 11 \\
& l=-15 \rightarrow 8
\end{aligned}
$$

3 standard reflections every 150 reflections intensity decay: $-0.4 \%$

Secondary atom site location: difference Fourier map

Hydrogen site location: inferred from neighbouring sites

$\mathrm{H}$-atom parameters constrained

$w=1 /\left[\sigma^{2}\left(F_{\mathrm{o}}^{2}\right)+(0.0433 P)^{2}+0.7479 P\right]$ where $P=\left(F_{\mathrm{o}}^{2}+2 F_{\mathrm{c}}^{2}\right) / 3$

$(\Delta / \sigma)_{\max }=0.001$

$\Delta \rho_{\max }=0.49$ e $\AA^{-3}$

$\Delta \rho_{\text {min }}=-0.53$ e $\AA^{-3}$ 


$\begin{array}{lllll}\text { C12 } & 0.70249(8) & 0.80052(15) & 0.61334(11) & 0.0140(3) \\ \text { C13 } & 0.64295(9) & 0.87459(16) & 0.53406(12) & 0.0176(3) \\ \text { C14 } & 0.66574(9) & 0.99175(17) & 0.46685(12) & 0.0208(3) \\ \text { C15 } & 0.74839(10) & 1.03185(17) & 0.47995(12) & 0.0210(3) \\ \text { C16 } & 0.89800(9) & 0.99932(17) & 0.57563(13) & 0.0212(3) \\ \text { C17 } & 0.95874(9) & 0.93028(17) & 0.65587(13) & 0.0219(3) \\ \text { C18 } & 0.93670(9) & 0.81701(16) & 0.72581(12) & 0.0193(3) \\ \text { C19 } & 0.85400(8) & 0.77372(15) & 0.71310(12) & 0.0157(3) \\ \text { C20 } & 0.78937(8) & 0.84224(14) & 0.63022(11) & 0.0133(3) \\ \text { C21 } & 0.81193(9) & 0.95853(16) & 0.56097(12) & 0.0170(3) \\ \text { H1B } & 0.4283 & 0.6476 & 0.8960 & 0.0207^{*} \\ \text { H2A } & 0.4672 & 0.8106 & 0.8850 & 0.0207^{*} \\ \text { H3 } & 0.2769 & 0.4334 & 0.5466 & 0.0179^{*} \\ \text { H4 } & 0.0879 & 0.6382 & 0.6603 & 0.0182^{*} \\ \text { H5 } & 0.5777 & 0.7643 & 0.8024 & 0.0199^{*} \\ \text { H6 } & 0.5617 & 0.5962 & 0.6100 & 0.0213^{*} \\ \text { H7A } & 0.7048 & 0.5791 & 0.6626 & 0.0209^{*} \\ \text { H8B } & 0.7033 & 0.6925 & 0.7651 & 0.0209^{*} \\ \text { H9 } & 0.5855 & 0.8470 & 0.5240 & 0.0211^{*} \\ \text { H10 } & 0.6236 & 1.0424 & 0.4127 & 0.0249^{*} \\ \text { H11 } & 0.7633 & 1.1098 & 0.4341 & 0.0252^{*} \\ \text { H12 } & 0.9135 & 1.0757 & 0.5290 & 0.0255^{*} \\ \text { H13 } & 1.0160 & 0.9588 & 0.6645 & 0.0263^{*} \\ \text { H14 } & 0.9791 & 0.7702 & 0.7820 & 0.0231^{*} \\ \text { H15 } & 0.8400 & 0.6968 & 0.7605 & 0.0188^{*}\end{array}$

Atomic displacement parameters $\left(\AA^{2}\right)$

\begin{tabular}{lllllll}
\hline & $U^{11}$ & $U^{22}$ & $U^{33}$ & $U^{12}$ & $U^{13}$ & $U^{23}$ \\
\hline C11 & $0.01563(16)$ & $0.0285(2)$ & $0.01929(17)$ & $-0.00243(13)$ & $0.00266(12)$ & $-0.00941(13)$ \\
C12 & $0.01504(16)$ & $0.01710(17)$ & $0.02549(18)$ & $-0.00033(12)$ & $0.00772(12)$ & $-0.00669(13)$ \\
O1 & $0.0124(5)$ & $0.0160(5)$ & $0.0241(5)$ & $0.0000(4)$ & $0.0036(4)$ & $-0.0044(4)$ \\
O2 & $0.0161(5)$ & $0.0212(5)$ & $0.0225(5)$ & $0.0025(4)$ & $0.0078(4)$ & $-0.0020(4)$ \\
N1 & $0.0120(6)$ & $0.0189(6)$ & $0.0228(6)$ & $-0.0021(5)$ & $0.0046(5)$ & $-0.0003(5)$ \\
C1 & $0.0116(6)$ & $0.0192(7)$ & $0.0204(7)$ & $0.0005(5)$ & $0.0020(5)$ & $-0.0002(6)$ \\
C2 & $0.0139(6)$ & $0.0148(7)$ & $0.0210(7)$ & $0.0018(5)$ & $0.0053(5)$ & $0.0031(6)$ \\
C3 & $0.0138(6)$ & $0.0135(6)$ & $0.0197(7)$ & $0.0027(5)$ & $0.0061(5)$ & $0.0043(5)$ \\
C4 & $0.0160(6)$ & $0.0162(6)$ & $0.0134(6)$ & $0.0024(5)$ & $0.0048(5)$ & $0.0004(5)$ \\
C5 & $0.0155(7)$ & $0.0170(7)$ & $0.0141(6)$ & $-0.0015(5)$ & $0.0024(5)$ & $0.0006(5)$ \\
C6 & $0.0125(6)$ & $0.0174(7)$ & $0.0165(6)$ & $0.0008(5)$ & $0.0051(5)$ & $0.0021(5)$ \\
C7 & $0.0155(6)$ & $0.0118(6)$ & $0.0164(6)$ & $0.0024(5)$ & $0.0068(5)$ & $0.0007(5)$ \\
C8 & $0.0137(6)$ & $0.0128(6)$ & $0.0162(6)$ & $-0.0001(5)$ & $0.0039(5)$ & $0.0022(5)$ \\
C9 & $0.0138(6)$ & $0.0150(7)$ & $0.0165(6)$ & $0.0018(5)$ & $0.0053(5)$ & $0.0033(5)$ \\
C10 & $0.0146(6)$ & $0.0142(6)$ & $0.0206(7)$ & $0.0000(5)$ & $0.0032(5)$ & $0.0037(6)$ \\
C11 & $0.0110(6)$ & $0.0173(7)$ & $0.0245(7)$ & $0.0012(5)$ & $0.0050(5)$ & $0.0044(6)$ \\
C12 & $0.0140(6)$ & $0.0132(6)$ & $0.0155(6)$ & $0.0001(5)$ & $0.0051(5)$ & $-0.0018(5)$ \\
C13 & $0.0162(7)$ & $0.0183(7)$ & $0.0177(7)$ & $0.0011(5)$ & $0.0021(5)$ & $-0.0014(6)$ \\
C14 & $0.0244(7)$ & $0.0205(7)$ & $0.0157(7)$ & $0.0033(6)$ & $0.0003(6)$ & $0.0017(6)$ \\
& & & & & &
\end{tabular}


supporting information

\begin{tabular}{lllllll}
$\mathrm{C} 15$ & $0.0282(8)$ & $0.0187(7)$ & $0.0172(7)$ & $-0.0015(6)$ & $0.0072(6)$ & $0.0027(6)$ \\
$\mathrm{C} 16$ & $0.0231(8)$ & $0.0187(7)$ & $0.0246(7)$ & $-0.0042(6)$ & $0.0110(6)$ & $0.0001(6)$ \\
$\mathrm{C} 17$ & $0.0153(7)$ & $0.0224(8)$ & $0.0298(8)$ & $-0.0038(6)$ & $0.0089(6)$ & $-0.0056(6)$ \\
$\mathrm{C} 18$ & $0.0150(7)$ & $0.0206(7)$ & $0.0219(7)$ & $0.0030(6)$ & $0.0035(6)$ & $-0.0033(6)$ \\
$\mathrm{C} 19$ & $0.0161(6)$ & $0.0143(6)$ & $0.0173(7)$ & $0.0004(5)$ & $0.0050(5)$ & $-0.0017(5)$ \\
$\mathrm{C} 20$ & $0.0144(6)$ & $0.0127(6)$ & $0.0137(6)$ & $-0.0000(5)$ & $0.0050(5)$ & $-0.0029(5)$ \\
$\mathrm{C} 21$ & $0.0199(7)$ & $0.0157(7)$ & $0.0169(7)$ & $-0.0008(6)$ & $0.0072(5)$ & $-0.0021(6)$ \\
\hline
\end{tabular}

Geometric parameters $\left(\AA,{ }^{\circ}\right)$

\begin{tabular}{|c|c|c|c|}
\hline $\mathrm{C} 11-\mathrm{C} 5$ & $1.7319(15)$ & $\mathrm{C} 15-\mathrm{C} 21$ & $1.415(2)$ \\
\hline $\mathrm{Cl} 2-\mathrm{C} 7$ & $1.7313(17)$ & $\mathrm{C} 16-\mathrm{C} 17$ & $1.367(2)$ \\
\hline $\mathrm{O} 1-\mathrm{C} 1$ & $1.4524(17)$ & $\mathrm{C} 16-\mathrm{C} 21$ & $1.421(3)$ \\
\hline $\mathrm{O} 1-\mathrm{C} 8$ & $1.3543(17)$ & $\mathrm{C} 17-\mathrm{C} 18$ & 1.409 (3) \\
\hline $\mathrm{O} 2-\mathrm{C} 3$ & $1.246(2)$ & $\mathrm{C} 18-\mathrm{C} 19$ & 1.377 (2) \\
\hline $\mathrm{N} 1-\mathrm{C} 10$ & $1.330(3)$ & $\mathrm{C} 19-\mathrm{C} 20$ & 1.4185 (19) \\
\hline $\mathrm{N} 1-\mathrm{C} 11$ & 1.4554 (19) & $\mathrm{C} 20-\mathrm{C} 21$ & $1.426(3)$ \\
\hline $\mathrm{C} 1-\mathrm{C} 2$ & $1.498(3)$ & $\mathrm{N} 1-\mathrm{H} 6$ & 0.880 \\
\hline $\mathrm{C} 2-\mathrm{C} 3$ & $1.430(2)$ & $\mathrm{C} 1-\mathrm{H} 1 \mathrm{~B}$ & 0.990 \\
\hline $\mathrm{C} 2-\mathrm{C} 10$ & $1.377(2)$ & $\mathrm{C} 1-\mathrm{H} 2 \mathrm{~A}$ & 0.990 \\
\hline $\mathrm{C} 3-\mathrm{C} 9$ & $1.496(2)$ & $\mathrm{C} 4-\mathrm{H} 3$ & 0.950 \\
\hline $\mathrm{C} 4-\mathrm{C} 5$ & $1.384(2)$ & $\mathrm{C} 6-\mathrm{H} 4$ & 0.950 \\
\hline $\mathrm{C} 4-\mathrm{C} 9$ & $1.3958(19)$ & $\mathrm{C} 10-\mathrm{H} 5$ & 0.950 \\
\hline $\mathrm{C} 5-\mathrm{C} 6$ & $1.391(2)$ & $\mathrm{C} 11-\mathrm{H} 7 \mathrm{~A}$ & 0.990 \\
\hline $\mathrm{C} 6-\mathrm{C} 7$ & $1.3817(19)$ & $\mathrm{C} 11-\mathrm{H} 8 \mathrm{~B}$ & 0.990 \\
\hline $\mathrm{C} 7-\mathrm{C} 8$ & $1.400(2)$ & $\mathrm{C} 13-\mathrm{H} 9$ & 0.950 \\
\hline $\mathrm{C} 8-\mathrm{C} 9$ & $1.403(2)$ & $\mathrm{C} 14-\mathrm{H} 10$ & 0.950 \\
\hline $\mathrm{C} 11-\mathrm{C} 12$ & $1.516(3)$ & C15-H11 & 0.950 \\
\hline $\mathrm{C} 12-\mathrm{C} 13$ & $1.3703(19)$ & $\mathrm{C} 16-\mathrm{H} 12$ & 0.950 \\
\hline $\mathrm{C} 12-\mathrm{C} 20$ & $1.434(2)$ & $\mathrm{C} 17-\mathrm{H} 13$ & 0.950 \\
\hline $\mathrm{C} 13-\mathrm{C} 14$ & $1.418(3)$ & $\mathrm{C} 18-\mathrm{H} 14$ & 0.950 \\
\hline $\mathrm{C} 14-\mathrm{C} 15$ & $1.368(3)$ & $\mathrm{C} 19-\mathrm{H} 15$ & 0.950 \\
\hline $\mathrm{Cl} 2 \cdots \mathrm{O} 1$ & $2.9030(16)$ & $\mathrm{C} 2 \cdots \mathrm{H} 9^{\text {iv }}$ & 3.5215 \\
\hline $\mathrm{O} 1 \cdots \mathrm{C} 3$ & $2.878(3)$ & $\mathrm{C} 2 \cdots \mathrm{H} 10^{\text {vi }}$ & 3.2427 \\
\hline $\mathrm{O} 2 \cdots \mathrm{N} 1$ & $2.7938(19)$ & $\mathrm{C} 2 \cdots \mathrm{H} 10^{\text {iv }}$ & 3.5375 \\
\hline $\mathrm{O} 2 \cdots \mathrm{C} 4$ & $2.844(2)$ & $\mathrm{C} 3 \cdots \mathrm{H} 2 \mathrm{~A}^{\mathrm{ii}}$ & 3.3458 \\
\hline $\mathrm{O} 2 \cdots \mathrm{C} 10$ & 2.889 (2) & $\mathrm{C} 3 \cdots \mathrm{H} 5^{\mathrm{ii}}$ & 2.9718 \\
\hline $\mathrm{N} 1 \cdots \mathrm{C} 3$ & $2.956(2)$ & $\mathrm{C} 3 \cdots \mathrm{H} 10^{\text {vi }}$ & 3.3648 \\
\hline $\mathrm{N} 1 \cdots \mathrm{C} 13$ & $2.846(3)$ & $\mathrm{C} 4 \cdots \mathrm{H} 5^{\mathrm{ii}}$ & 3.4301 \\
\hline $\mathrm{C} 1 \cdots \mathrm{C} 9$ & $2.755(3)$ & $\mathrm{C} 4 \cdots \mathrm{H} 7 \mathrm{~A}^{\mathrm{iii}}$ & 3.3561 \\
\hline $\mathrm{C} 2 \cdots \mathrm{C} 8$ & $2.794(3)$ & $\mathrm{C} 4 \cdots \mathrm{H} 8 \mathrm{~B}^{\mathrm{ii}}$ & 3.2427 \\
\hline $\mathrm{C} 4 \cdots \mathrm{C} 7$ & $2.775(3)$ & $\mathrm{C} 4 \cdots \mathrm{H} 11^{\mathrm{vi}}$ & 3.4591 \\
\hline $\mathrm{C} 5 \cdots \mathrm{C} 8$ & $2.780(3)$ & $\mathrm{C} 5 \cdots \mathrm{H} 11^{\mathrm{vi}}$ & 3.4793 \\
\hline $\mathrm{C} 6 \cdots \mathrm{C} 9$ & $2.800(3)$ & $\mathrm{C} 5 \cdots \mathrm{H} 15^{\mathrm{ii}}$ & 3.4109 \\
\hline $\mathrm{C} 10 \cdots \mathrm{C} 12$ & $3.370(3)$ & $\mathrm{C} 6 \cdots \mathrm{H} 11^{\mathrm{vi}}$ & 3.1293 \\
\hline $\mathrm{C} 10 \cdots \mathrm{C} 13$ & $3.583(3)$ & $\mathrm{C} 6 \cdots \mathrm{H} 12^{\mathrm{vi}}$ & 3.5089 \\
\hline $\mathrm{C} 11 \cdots \mathrm{C} 19$ & $2.937(3)$ & $\mathrm{C} 6 \cdots \mathrm{H} 14^{\text {viii }}$ & 3.5789 \\
\hline
\end{tabular}




\begin{tabular}{|c|c|c|c|}
\hline $\mathrm{C} 12 \cdots \mathrm{C} 15$ & $2.810(3)$ & $\mathrm{C} 7 \cdots \mathrm{H} 11^{\mathrm{vi}}$ & 2.7047 \\
\hline $\mathrm{C} 13 \cdots \mathrm{C} 21$ & $2.801(3)$ & $\mathrm{C} 8 \cdots \mathrm{H}_{7} \mathrm{~A}^{\mathrm{v}}$ & 3.5798 \\
\hline $\mathrm{C} 14 \cdots \mathrm{C} 20$ & $2.819(3)$ & $\mathrm{C} 8 \cdots \mathrm{H} 10^{\mathrm{vi}}$ & 3.4120 \\
\hline $\mathrm{C} 16 \cdots \mathrm{C} 19$ & $2.792(3)$ & $\mathrm{C} 8 \cdots \mathrm{H} 11^{\mathrm{vi}}$ & 2.6837 \\
\hline $\mathrm{C} 17 \cdots \mathrm{C} 20$ & $2.819(3)$ & $\mathrm{C} 9 \cdots \mathrm{H} 5^{\mathrm{ii}}$ & 3.3815 \\
\hline $\mathrm{C} 18 \cdots \mathrm{C} 21$ & $2.806(3)$ & $\mathrm{C} 9 \cdots \mathrm{H} 10^{\text {vi }}$ & 3.5560 \\
\hline $\mathrm{Cl} 1 \cdots \mathrm{Cl} 2^{\mathrm{i}}$ & $3.4590(17)$ & $\mathrm{C} 9 \cdots \mathrm{H} 11^{\mathrm{vi}}$ & 3.0717 \\
\hline $\mathrm{C} 11 \cdots \mathrm{C} 18^{\mathrm{ii}}$ & $3.531(3)$ & $\mathrm{C} 10 \cdots \mathrm{H} 9^{\text {iv }}$ & 3.3197 \\
\hline $\mathrm{C} 11 \cdots \mathrm{C} 18^{\mathrm{iii}}$ & $3.372(3)$ & $\mathrm{C} 10 \cdots \mathrm{H} 10^{\text {iv }}$ & 3.0585 \\
\hline $\mathrm{C} 11 \cdots \mathrm{C} 19^{\mathrm{ii}}$ & $3.531(3)$ & $\mathrm{C} 11 \cdots \mathrm{H} 3^{\mathrm{iii}}$ & 3.1482 \\
\hline $\mathrm{C} 11 \cdots \mathrm{C} 19^{\mathrm{iii}}$ & $3.298(3)$ & $\mathrm{C} 12 \cdots \mathrm{H} 3^{\mathrm{iii}}$ & 2.9038 \\
\hline $\mathrm{C} 11 \cdots \mathrm{C} 20^{\mathrm{iii}}$ & $3.483(2)$ & $\mathrm{C} 13 \cdots \mathrm{H}_{1 B^{\mathrm{v}}}$ & 2.8981 \\
\hline $\mathrm{Cl} 2 \cdots \mathrm{Cl}^{\text {iv }}$ & $3.4590(17)$ & $\mathrm{C} 13 \cdots \mathrm{H} 1 \mathrm{~B}^{\mathrm{i}}$ & 3.5306 \\
\hline $\mathrm{Cl} 2 \cdots \mathrm{C}^{\text {iv }}$ & $3.544(3)$ & $\mathrm{C} 13 \cdots \mathrm{H} 2 \mathrm{~A}^{\mathrm{i}}$ & 3.4463 \\
\hline $\mathrm{Cl} 2 \cdots \mathrm{C} 5^{\text {iv }}$ & $3.478(3)$ & $\mathrm{C} 13 \cdots \mathrm{H} 3^{\mathrm{iii}}$ & 3.2759 \\
\hline $\mathrm{O} 1 \cdots \mathrm{N} 1^{\mathrm{v}}$ & $3.327(3)$ & $\mathrm{C} 13 \cdots \mathrm{H} 5^{\mathrm{i}}$ & 3.0174 \\
\hline $\mathrm{O} 1 \cdots \mathrm{C} 11^{\mathrm{v}}$ & $3.401(3)$ & $\mathrm{C} 14 \cdots \mathrm{H}_{1} \mathrm{~B}^{\mathrm{v}}$ & 2.8448 \\
\hline $\mathrm{O} 2 \cdots \mathrm{O} 2^{\mathrm{iii}}$ & $3.285(3)$ & $\mathrm{C} 14 \cdots \mathrm{H} 5^{\mathrm{i}}$ & 3.1497 \\
\hline $\mathrm{O} 2 \cdots \mathrm{N} 1^{\mathrm{iii}}$ & $3.306(3)$ & $\mathrm{C} 14 \cdots \mathrm{H}_{8} \mathrm{~B}^{\mathrm{i}}$ & 3.0966 \\
\hline $\mathrm{O} 2 \cdots \mathrm{C} 1^{\mathrm{ii}}$ & $3.472(3)$ & $\mathrm{C} 15 \cdots \mathrm{H} 8 \mathrm{~B}^{\mathrm{i}}$ & 3.2195 \\
\hline $\mathrm{O} 2 \cdots \mathrm{C} 1^{\mathrm{i}}$ & $3.542(3)$ & $\mathrm{C} 16 \cdots \mathrm{H} 4^{\mathrm{v}}$ & 3.3653 \\
\hline $\mathrm{O} 2 \cdots \mathrm{C} 10^{\mathrm{ii}}$ & $3.567(3)$ & $\mathrm{C} 16 \cdots \mathrm{H} 13^{\mathrm{ix}}$ & 3.4908 \\
\hline $\mathrm{N} 1 \cdots \mathrm{O} 1^{\mathrm{ii}}$ & $3.327(3)$ & $\mathrm{C} 16 \cdots \mathrm{H} 14^{\mathrm{x}}$ & 3.3549 \\
\hline $\mathrm{N} 1 \cdots \mathrm{O} 2^{\mathrm{iii}}$ & $3.306(3)$ & $\mathrm{C} 17 \cdots \mathrm{H} 4^{\mathrm{xi}}$ & 3.3395 \\
\hline $\mathrm{C} 1 \cdots \mathrm{O} 2^{\mathrm{v}}$ & $3.472(3)$ & $\mathrm{C} 17 \cdots \mathrm{H}_{4}{ }^{\mathrm{v}}$ & 3.1019 \\
\hline $\mathrm{C} 1 \cdots \mathrm{O} 2^{\mathrm{iv}}$ & $3.542(3)$ & $\mathrm{C} 17 \cdots \mathrm{H} 12^{\mathrm{ix}}$ & 3.3585 \\
\hline $\mathrm{C} 4 \cdots \mathrm{Cl} 2^{\mathrm{i}}$ & $3.544(3)$ & $\mathrm{C} 17 \cdots \mathrm{H} 14^{\mathrm{x}}$ & 3.2288 \\
\hline $\mathrm{C} 5 \cdots \mathrm{Cl} 2^{\mathrm{i}}$ & $3.478(3)$ & $\mathrm{C} 18 \cdots \mathrm{H} 4^{\mathrm{xi}}$ & 3.1738 \\
\hline $\mathrm{C} 5 \cdots \mathrm{C} 19^{\mathrm{ii}}$ & $3.302(3)$ & $\mathrm{C} 18 \cdots \mathrm{H} 4^{\mathrm{v}}$ & 3.2351 \\
\hline $\mathrm{C} 6{ }^{\cdots} \mathrm{C} 16^{\mathrm{ii}}$ & $3.558(3)$ & $\mathrm{C} 18 \cdots \mathrm{H} 13^{\mathrm{xii}}$ & 3.4745 \\
\hline $\mathrm{C} 6{ }^{\cdots} \mathrm{C} 17^{\mathrm{ii}}$ & $3.515(3)$ & $\mathrm{C} 20 \cdots \mathrm{H} 3^{\mathrm{iii}}$ & 3.2717 \\
\hline C6 $\cdots \mathrm{C} 18^{\mathrm{ii}}$ & $3.466(3)$ & $\mathrm{H} 1 \mathrm{~B} \cdots \mathrm{C} 13^{\mathrm{ii}}$ & 2.8981 \\
\hline $\mathrm{C} 6 \cdots \mathrm{C} 19^{\mathrm{ii}}$ & $3.479(3)$ & $\mathrm{H} 1 \mathrm{~B} \cdots \mathrm{C} 13^{\mathrm{iv}}$ & 3.5306 \\
\hline $\mathrm{C} 6{ }^{\cdots} \mathrm{C} 20^{\mathrm{ii}}$ & $3.542(3)$ & $\mathrm{H} 1 \mathrm{~B} \cdots \mathrm{C} 14^{\mathrm{ii}}$ & 2.8448 \\
\hline $\mathrm{C} 6{ }^{\cdots} \mathrm{C} 21^{\mathrm{ii}}$ & $3.566(3)$ & $\mathrm{H} 1 \mathrm{~B} \cdots \mathrm{H}^{\mathrm{ii}}$ & 2.8715 \\
\hline $\mathrm{C} 7 \cdots \mathrm{C} 21^{\mathrm{ii}}$ & 3.399 (3) & $\mathrm{H} 1 \mathrm{~B} \cdots \mathrm{H} 9^{\mathrm{iv}}$ & 2.6810 \\
\hline $\mathrm{C} 8 \cdots \mathrm{C} 15^{\mathrm{vi}}$ & $3.511(3)$ & $\mathrm{H} 1 \mathrm{~B} \cdots \mathrm{H} 10^{\mathrm{ii}}$ & 2.7785 \\
\hline $\mathrm{C} 10 \cdots \mathrm{O} 2^{\mathrm{v}}$ & $3.567(3)$ & $\mathrm{H} 1 \mathrm{~B} \cdots \mathrm{H} 10^{\mathrm{iv}}$ & 3.5696 \\
\hline $\mathrm{C} 10 \cdots \mathrm{C} 13^{\text {iv }}$ & $3.567(3)$ & $\mathrm{H} 2 \mathrm{~A} \cdots \mathrm{O} 2^{v}$ & 2.5248 \\
\hline $\mathrm{C} 10 \cdots \mathrm{C} 14^{\mathrm{iv}}$ & $3.418(3)$ & $\mathrm{H} 2 \mathrm{~A} \cdots \mathrm{O} 2^{\mathrm{iv}}$ & 2.9081 \\
\hline $\mathrm{C} 11 \cdots \mathrm{O} 1^{\mathrm{ii}}$ & $3.401(3)$ & $\mathrm{H} 2 \mathrm{~A} \cdots \mathrm{N} 1^{\mathrm{v}}$ & 3.1678 \\
\hline $\mathrm{C} 13 \cdots \mathrm{C} 10^{\mathrm{i}}$ & $3.567(3)$ & $\mathrm{H} 2 \mathrm{~A} \cdots \mathrm{N} 1^{\text {iv }}$ & 3.5686 \\
\hline $\mathrm{C} 14 \cdots \mathrm{C} 10^{\mathrm{i}}$ & $3.418(3)$ & $\mathrm{H} 2 \mathrm{~A} \cdots \mathrm{C} 3^{\mathrm{v}}$ & 3.3458 \\
\hline $\mathrm{C} 15 \cdots \mathrm{C} 8^{\mathrm{vi}}$ & $3.511(3)$ & $\mathrm{H} 2 \mathrm{~A} \cdots \mathrm{C} 13^{\mathrm{iv}}$ & 3.4463 \\
\hline $\mathrm{C} 16{ }^{\cdots} \mathrm{C} 6^{\mathrm{v}}$ & $3.558(3)$ & $\mathrm{H} 2 \mathrm{~A} \cdots \mathrm{H} 6^{\mathrm{v}}$ & 2.5906 \\
\hline $\mathrm{C} 17 \cdots \mathrm{C} 6^{\mathrm{v}}$ & $3.515(3)$ & $\mathrm{H} 2 \mathrm{~A} \cdots \mathrm{H} 6^{\mathrm{iv}}$ & 2.9221 \\
\hline $\mathrm{C} 18 \cdots \mathrm{Cl}^{\mathrm{v}}$ & $3.531(3)$ & $\mathrm{H} 2 \mathrm{~A} \cdots \mathrm{H} 9^{\text {iv }}$ & 2.6567 \\
\hline $\mathrm{C} 18 \cdots \mathrm{Cl} 1^{\mathrm{iii}}$ & $3.372(3)$ & $\mathrm{H} 3 \cdots \mathrm{Cl} 2^{\mathrm{i}}$ & 3.4819 \\
\hline
\end{tabular}




\begin{tabular}{|c|c|c|c|}
\hline $\mathrm{C} 18 \cdots \mathrm{C}^{\mathrm{v}}$ & $3.466(3)$ & $\mathrm{H} 3 \cdots \mathrm{C} 11^{\mathrm{iii}}$ & 3.1482 \\
\hline $\mathrm{C} 19 \cdots \mathrm{Cl1}^{\mathrm{v}}$ & $3.531(3)$ & $\mathrm{H} 3{ }^{\cdots} \cdot \mathrm{C} 12^{\mathrm{iii}}$ & 2.9038 \\
\hline $\mathrm{C} 19 \cdots \mathrm{Cl} 1^{\mathrm{iii}}$ & $3.298(3)$ & $\mathrm{H} 3 \cdots \mathrm{C} 13^{\mathrm{iii}}$ & 3.2759 \\
\hline $\mathrm{C} 19 \cdots \mathrm{C}^{\mathrm{v}}$ & $3.302(3)$ & $\mathrm{H} 3 \cdots \mathrm{C} 20^{\mathrm{iii}}$ & 3.2717 \\
\hline $\mathrm{C} 19 \cdots \mathrm{C} 6^{\mathrm{v}}$ & $3.479(3)$ & $\mathrm{H} 3 \cdots \mathrm{H} 5^{\mathrm{ii}}$ & 3.0475 \\
\hline $\mathrm{C} 20 \cdots \mathrm{C} 11^{\mathrm{iii}}$ & $3.483(2)$ & $\mathrm{H} 3 \cdots \mathrm{H} 6^{\mathrm{iii}}$ & 3.5623 \\
\hline $\mathrm{C} 20 \cdots \mathrm{C} 6^{\mathrm{v}}$ & $3.542(3)$ & $\mathrm{H} 3 \cdots \mathrm{H} 7 \mathrm{~A}^{\mathrm{iii}}$ & 2.5963 \\
\hline $\mathrm{C} 21 \cdots \mathrm{C} 6^{\mathrm{v}}$ & $3.566(3)$ & $\mathrm{H} 3 \cdots{ }^{\prime}{ }^{3} \mathrm{~B}^{\mathrm{ii}}$ & 3.0827 \\
\hline $\mathrm{C} 21 \cdots \mathrm{C} 7^{\mathrm{v}}$ & $3.399(3)$ & $\mathrm{H} 3 \cdots \mathrm{H} 9^{\mathrm{iii}}$ & 3.5808 \\
\hline $\mathrm{C} 11 \cdots \mathrm{H} 3$ & 2.8044 & $\mathrm{H} 4 \cdots \mathrm{Cl} 1^{\mathrm{vii}}$ & 3.3003 \\
\hline $\mathrm{Cl} 1 \cdots \mathrm{H} 4$ & 2.8011 & $\mathrm{H} 4 \cdots \mathrm{C} 16^{\mathrm{ii}}$ & 3.3653 \\
\hline $\mathrm{Cl} 2 \cdots \mathrm{H} 4$ & 2.8007 & $\mathrm{H} 4 \cdots \mathrm{C} 17^{\text {viii }}$ & 3.3395 \\
\hline $\mathrm{O} 2 \cdots \mathrm{H} 3$ & 2.5793 & $\mathrm{H} 4 \cdots \mathrm{C} 17^{\mathrm{ii}}$ & 3.1019 \\
\hline $\mathrm{O} 2 \cdots \mathrm{H} 6$ & 2.1816 & $\mathrm{H} 4 \cdots \mathrm{C} 18^{\mathrm{viii}}$ & 3.1738 \\
\hline $\mathrm{N} 1 \cdots \mathrm{H} 9$ & 2.4771 & $\mathrm{H} 4 \cdots \mathrm{C} 18^{\mathrm{ii}}$ & 3.2351 \\
\hline $\mathrm{C} 1 \cdots \mathrm{H} 5$ & 2.5838 & $\mathrm{H} 4 \cdots \mathrm{H} 12^{\mathrm{vi}}$ & 3.4114 \\
\hline $\mathrm{C} 2 \cdots \mathrm{H} 6$ & 2.6121 & $\mathrm{H} 4 \cdots \mathrm{H} 13^{\text {viii }}$ & 3.0915 \\
\hline $\mathrm{C} 3 \cdots \mathrm{H} 1 \mathrm{~B}$ & 2.8456 & $\mathrm{H} 4 \cdots \mathrm{H} 13^{\mathrm{ii}}$ & 3.3779 \\
\hline $\mathrm{C} 3 \cdots \mathrm{H} 2 \mathrm{~A}$ & 3.3086 & $\mathrm{H} 4 \cdots \mathrm{H} 14^{\mathrm{viii}}$ & 2.7925 \\
\hline $\mathrm{C} 3 \cdots \mathrm{H} 3$ & 2.6730 & $\mathrm{H} 4 \cdots \cdot \mathrm{H} 14^{\mathrm{ii}}$ & 3.5715 \\
\hline $\mathrm{C} 3 \cdots \mathrm{H} 5$ & 3.2932 & $\mathrm{H} 5 \cdots \mathrm{O} 2^{\mathrm{v}}$ & 2.7607 \\
\hline $\mathrm{C} 3 \cdots \mathrm{H} 6$ & 2.6854 & $\mathrm{H} 5 \cdots \mathrm{C} 3^{v}$ & 2.9718 \\
\hline $\mathrm{C} 4 \cdots \mathrm{H} 4$ & 3.2775 & $\mathrm{H} 5 \cdots \mathrm{C}^{\mathrm{v}}$ & 3.4301 \\
\hline $\mathrm{C} 6 \cdots \mathrm{H} 3$ & 3.2754 & $\mathrm{H} 5 \cdots \mathrm{C} 9^{v}$ & 3.3815 \\
\hline C $8 \cdots \mathrm{H} 1 \mathrm{~B}$ & 2.6266 & $\mathrm{H} 5 \cdots \mathrm{C} 13^{\text {iv }}$ & 3.0174 \\
\hline $\mathrm{C} 8 \cdots \mathrm{H} 2 \mathrm{~A}$ & 3.1711 & $\mathrm{H} 5 \cdots \mathrm{C} 14^{\mathrm{iv}}$ & 3.1497 \\
\hline $\mathrm{C} 8 \cdots \mathrm{H} 3$ & 3.2817 & $\mathrm{H} 5 \cdots \mathrm{H} 3^{\mathrm{v}}$ & 3.0475 \\
\hline $\mathrm{C} 8 \cdots \mathrm{H} 4$ & 3.2853 & $\mathrm{H} 5 \cdots \mathrm{H} 9^{\text {iv }}$ & 2.8161 \\
\hline C9 $\cdots \mathrm{H} 1 \mathrm{~B}$ & 2.9757 & $\mathrm{H} 5 \cdots \mathrm{H} 10^{\mathrm{iv}}$ & 3.0576 \\
\hline $\mathrm{C} 10 \cdots \mathrm{H} 1 \mathrm{~B}$ & 2.9791 & $\mathrm{H} 6 \cdots \mathrm{O} 1^{\mathrm{ii}}$ & 3.1444 \\
\hline $\mathrm{C} 10 \cdots \mathrm{H} 2 \mathrm{~A}$ & 2.5432 & $\mathrm{H} 6 \cdots \mathrm{O} 2^{\mathrm{iii}}$ & 2.5402 \\
\hline $\mathrm{C} 10 \cdots \mathrm{H} 7 \mathrm{~A}$ & 3.1414 & $\mathrm{H} 6 \cdots \mathrm{C} 1^{\mathrm{ii}}$ & 3.2859 \\
\hline $\mathrm{C} 10 \cdots \mathrm{H} 8 \mathrm{~B}$ & 2.5054 & $\mathrm{H} 6 \cdots \mathrm{H} 2 \mathrm{~A}^{\mathrm{ii}}$ & 2.5906 \\
\hline $\mathrm{C} 10 \cdots \mathrm{H} 9$ & 3.1166 & $\mathrm{H} 6 \cdots \mathrm{H} 2 \mathrm{~A}^{\mathrm{i}}$ & 2.9221 \\
\hline 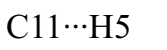 & 2.5417 & $\mathrm{H} 6 \cdots \mathrm{H} 3^{\mathrm{iii}}$ & 3.5623 \\
\hline $\mathrm{C} 11 \cdots \mathrm{H} 9$ & 2.6689 & $\mathrm{H} 6 \cdots \mathrm{H}^{\mathrm{iii}}$ & 3.4103 \\
\hline $\mathrm{C} 11 \cdots \mathrm{H} 15$ & 2.5932 & $\mathrm{H} 7 \mathrm{~A} \cdots \mathrm{Cl} 2^{\mathrm{ii}}$ & 2.8708 \\
\hline $\mathrm{C} 12 \cdots \mathrm{H} 5$ & 3.3778 & $\mathrm{H} 7 \mathrm{~A} \cdots \mathrm{O} 1^{\mathrm{ii}}$ & 2.7154 \\
\hline 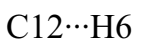 & 2.9216 & $\mathrm{H} 7 \mathrm{~A} \cdots \mathrm{O} 2^{\mathrm{iii}}$ & 3.3604 \\
\hline $\mathrm{C} 12 \cdots \mathrm{H} 10$ & 3.2782 & $\mathrm{H} 7 \mathrm{~A} \cdots \mathrm{C} 4^{\mathrm{iii}}$ & 3.3561 \\
\hline $\mathrm{C} 12 \cdots \mathrm{H} 15$ & 2.6946 & $\mathrm{H} 7 \mathrm{~A} \cdots \mathrm{C} 8^{\mathrm{ii}}$ & 3.5798 \\
\hline 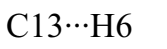 & 3.0440 & $\mathrm{H} 7 \mathrm{~A} \cdots \mathrm{H} 3^{\mathrm{iii}}$ & 2.5963 \\
\hline $\mathrm{C} 13 \cdots \mathrm{H} 7 \mathrm{~A}$ & 3.1064 & $\mathrm{H} 8 \mathrm{~B} \cdots \mathrm{C} 4^{\mathrm{v}}$ & 3.2427 \\
\hline C13 $\cdots$ H8B & 3.1775 & $\mathrm{H} 8 \mathrm{~B} \cdots \mathrm{C} 14^{\mathrm{iv}}$ & 3.0966 \\
\hline $\mathrm{C} 13 \cdots \mathrm{H} 11$ & 3.2717 & $\mathrm{H} 8 \mathrm{~B} \cdots \mathrm{C} 15^{\mathrm{iv}}$ & 3.2195 \\
\hline $\mathrm{C} 15 \cdots \mathrm{H} 9$ & 3.2633 & $\mathrm{H} 8 \mathrm{~B} \cdots \mathrm{H}^{\mathrm{v}}$ & 3.0827 \\
\hline $\mathrm{C} 15 \cdots \mathrm{H} 12$ & 2.6531 & $\mathrm{H} 8 \mathrm{~B} \cdots \mathrm{H} 10^{\mathrm{iv}}$ & 3.1963 \\
\hline $\mathrm{C} 16 \cdots \mathrm{H} 11$ & 2.6523 & $\mathrm{H} 8 \mathrm{~B} \cdots \mathrm{H} 11^{\mathrm{iv}}$ & 3.3862 \\
\hline
\end{tabular}




\begin{tabular}{|c|c|}
\hline $\mathrm{C} 16 \cdots \mathrm{H} 14$ & 3.2588 \\
\hline $\mathrm{C} 17 \cdots \mathrm{H} 15$ & 3.2725 \\
\hline $\mathrm{C} 18 \cdots \mathrm{H} 12$ & 3.2633 \\
\hline C19 $\cdots$ H7A & 2.9380 \\
\hline 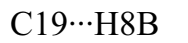 & 2.7588 \\
\hline 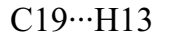 & 3.2701 \\
\hline $\mathrm{C} 20 \cdots \mathrm{H} 7 \mathrm{~A}$ & 2.7891 \\
\hline $\mathrm{C} 20 \cdots \mathrm{H} 8 \mathrm{~B}$ & 2.7134 \\
\hline $\mathrm{C} 20 \cdots \mathrm{H} 9$ & 3.2869 \\
\hline $\mathrm{C} 20 \cdots \mathrm{H} 11$ & 3.3119 \\
\hline $\mathrm{C} 20 \cdots \mathrm{H} 12$ & 3.3119 \\
\hline $\mathrm{C} 20 \cdots \mathrm{H} 14$ & 3.2890 \\
\hline $\mathrm{C} 21 \cdots \mathrm{H} 10$ & 3.2781 \\
\hline $\mathrm{C} 21 \cdots \mathrm{H} 13$ & 3.2836 \\
\hline $\mathrm{C} 21 \cdots \mathrm{H} 15$ & 3.3029 \\
\hline H1B $\cdots$ H5 & 3.0755 \\
\hline $\mathrm{H} 2 \mathrm{~A} \cdots \mathrm{H} 5$ & 2.2747 \\
\hline $\mathrm{H} 5 \cdots \mathrm{H} 6$ & 2.7173 \\
\hline $\mathrm{H} 5 \cdots \mathrm{H} 7 \mathrm{~A}$ & 3.3695 \\
\hline H5 $\cdots H 8 B$ & 2.2788 \\
\hline $\mathrm{H} 5 \cdots \mathrm{H} 9$ & 3.4527 \\
\hline H6 $\cdots$ H7A & 2.2832 \\
\hline H6 $\cdots$ H8B & 2.7639 \\
\hline H6 $\cdots \mathrm{H} 9$ & 2.5271 \\
\hline H7A $\cdots$ H9 & 3.2912 \\
\hline H7A $\cdots \mathrm{H} 15$ & 2.4895 \\
\hline H8B $\cdots \mathrm{H} 9$ & 3.3945 \\
\hline 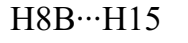 & 2.2403 \\
\hline $\mathrm{H} 9 \cdots \mathrm{H} 10$ & 2.3596 \\
\hline H10 $\cdots \mathrm{H} 11$ & 2.3125 \\
\hline $\mathrm{H} 11 \cdots \mathrm{H} 12$ & 2.4808 \\
\hline $\mathrm{H} 12 \cdots \mathrm{H} 13$ & 2.3085 \\
\hline $\mathrm{H} 13 \cdots \mathrm{H} 14$ & 2.3547 \\
\hline H14 $\cdots \mathrm{H} 15$ & 2.3174 \\
\hline $\mathrm{Cl} 1 \cdots \mathrm{H} 4^{\mathrm{vii}}$ & 3.3003 \\
\hline $\mathrm{C} 11 \cdots \mathrm{H} 14^{\mathrm{ii}}$ & 3.3665 \\
\hline $\mathrm{C} 11 \cdots \mathrm{H} 15^{\mathrm{ii}}$ & 3.3698 \\
\hline $\mathrm{Cl} 2 \cdots \mathrm{H} 3^{\text {iv }}$ & 3.4819 \\
\hline $\mathrm{Cl} 2 \cdots \mathrm{H} 7 \mathrm{~A}^{\mathrm{v}}$ & 2.8708 \\
\hline $\mathrm{Cl} 2 \cdots \mathrm{H} 11^{\mathrm{ii}}$ & 3.5822 \\
\hline $\mathrm{Cl} 2 \cdots \mathrm{H} 11^{\mathrm{vi}}$ & 3.4771 \\
\hline $\mathrm{Cl} 2 \cdots \mathrm{H} 12^{\mathrm{ii}}$ & 3.4716 \\
\hline $\mathrm{Cl} 2 \cdots \mathrm{H} 13^{\text {viii }}$ & 2.9779 \\
\hline $\mathrm{Cl} 2 \cdots \mathrm{H} 14^{\mathrm{viii}}$ & 3.1376 \\
\hline $\mathrm{Cl} 2 \cdots \mathrm{H} 15^{\mathrm{v}}$ & 3.2700 \\
\hline $\mathrm{O} 1 \cdots \mathrm{H} 6^{\mathrm{v}}$ & 3.1444 \\
\hline $\mathrm{O} 1 \cdots \mathrm{H}^{2} \mathrm{~A}^{\mathrm{v}}$ & 2.7154 \\
\hline
\end{tabular}

\begin{tabular}{|c|c|}
\hline $\mathrm{H} 9 \cdots \mathrm{O} 2^{\mathrm{iii}}$ & 3.5379 \\
\hline $\mathrm{H} 9 \cdots \mathrm{C} 1^{\mathrm{i}}$ & 3.0434 \\
\hline $\mathrm{H} 9 \cdots \mathrm{C} 2^{\mathrm{i}}$ & 3.5215 \\
\hline $\mathrm{H} 9 \cdots \mathrm{C} 10^{\mathrm{i}}$ & 3.3197 \\
\hline $\mathrm{H} 9 \cdots \mathrm{H} \mathrm{B}^{\mathrm{v}}$ & 2.8715 \\
\hline $\mathrm{H} 9 \cdots \mathrm{H} \mathrm{B}^{\mathrm{i}}$ & 2.6810 \\
\hline $\mathrm{H} 9 \cdots \mathrm{H} 2 \mathrm{~A}^{\mathrm{i}}$ & 2.6567 \\
\hline $\mathrm{H} 9 \cdots \mathrm{H} 3^{\mathrm{iii}}$ & 3.5808 \\
\hline $\mathrm{H} 9 \cdots \mathrm{H} 5^{\mathrm{i}}$ & 2.8161 \\
\hline $\mathrm{H} 10 \cdots \mathrm{O} 1^{\mathrm{vi}}$ & 3.1583 \\
\hline $\mathrm{H} 10 \cdots \mathrm{N} 1^{\mathrm{i}}$ & 3.3222 \\
\hline $\mathrm{H} 10 \cdots \mathrm{C} 2^{\mathrm{vi}}$ & 3.2427 \\
\hline $\mathrm{H} 10 \cdots \mathrm{C} 2^{\mathrm{i}}$ & 3.5375 \\
\hline $\mathrm{H} 10 \cdots \mathrm{C} 3^{\mathrm{vi}}$ & 3.3648 \\
\hline $\mathrm{H} 10 \cdots \mathrm{C} 8^{\mathrm{vi}}$ & 3.4120 \\
\hline $\mathrm{H} 10 \cdots \mathrm{C} 9^{\mathrm{vi}}$ & 3.5560 \\
\hline $\mathrm{H} 10 \cdots \mathrm{C} 10^{\mathrm{i}}$ & 3.0585 \\
\hline $\mathrm{H} 10 \cdots \mathrm{H} 1 \mathrm{~B}^{\mathrm{v}}$ & 2.7785 \\
\hline $\mathrm{H} 10 \cdots \mathrm{H} 1 \mathrm{~B}^{\mathrm{i}}$ & 3.5696 \\
\hline $\mathrm{H} 10 \cdots \mathrm{H} 5^{\mathrm{i}}$ & 3.0576 \\
\hline $\mathrm{H} 10 \cdots \mathrm{H} 8 \mathrm{~B}^{\mathrm{i}}$ & 3.1963 \\
\hline $\mathrm{H} 11 \cdots \mathrm{Cl} 2^{\mathrm{v}}$ & 3.5822 \\
\hline $\mathrm{H} 11 \cdots \mathrm{Cl} 2^{\mathrm{vi}}$ & 3.4771 \\
\hline $\mathrm{H} 11 \cdots \mathrm{O} 1^{\mathrm{vi}}$ & 3.1669 \\
\hline $\mathrm{H} 11 \cdots \mathrm{C} 4^{\mathrm{vi}}$ & 3.4591 \\
\hline $\mathrm{H} 11 \cdots \mathrm{C} 5^{\mathrm{vi}}$ & 3.4793 \\
\hline $\mathrm{H} 11 \cdots \mathrm{C}^{\mathrm{vi}}$ & 3.1293 \\
\hline $\mathrm{H} 11 \cdots \mathrm{C} 7^{\mathrm{vi}}$ & 2.7047 \\
\hline $\mathrm{H} 11 \cdots \mathrm{C} 8^{\mathrm{vi}}$ & 2.6837 \\
\hline $\mathrm{H} 11 \cdots \mathrm{C} 9^{\mathrm{vi}}$ & 3.0717 \\
\hline $\mathrm{H} 11 \cdots \mathrm{H} 8 \mathrm{~B}^{\mathrm{i}}$ & 3.3862 \\
\hline $\mathrm{H} 12 \cdots \mathrm{Cl} 2^{v}$ & 3.4716 \\
\hline $\mathrm{H} 12 \cdots \mathrm{C} 6^{\mathrm{vi}}$ & 3.5089 \\
\hline $\mathrm{H} 12 \cdots \mathrm{C} 17^{\mathrm{ix}}$ & 3.3585 \\
\hline $\mathrm{H} 12 \cdots \mathrm{H} 4^{\mathrm{vi}}$ & 3.4114 \\
\hline $\mathrm{H} 12 \cdots \mathrm{H} 12^{\mathrm{ix}}$ & 3.3307 \\
\hline $\mathrm{H} 12 \cdots \mathrm{H} 13^{\text {ix }}$ & 2.8245 \\
\hline $\mathrm{H} 12 \cdots \mathrm{H} 14^{\mathrm{x}}$ & 3.0781 \\
\hline $\mathrm{H} 13 \cdots \mathrm{Cl} 2^{\mathrm{xi}}$ & 2.9779 \\
\hline $\mathrm{H} 13{ }^{\cdots} \mathrm{C} 16^{\mathrm{ix}}$ & 3.4908 \\
\hline $\mathrm{H} 13 \cdots \mathrm{C} 18^{\mathrm{x}}$ & 3.4745 \\
\hline $\mathrm{H} 13 \cdots \mathrm{H} 4^{\mathrm{xi}}$ & 3.0915 \\
\hline $\mathrm{H} 13 \cdots \mathrm{H} 4^{v}$ & 3.3779 \\
\hline $\mathrm{H} 13 \cdots \mathrm{H} 12^{\mathrm{ix}}$ & 2.8245 \\
\hline $\mathrm{H} 13 \cdots \mathrm{H} 14^{\mathrm{x}}$ & 2.8458 \\
\hline $\mathrm{H} 13 \cdots \mathrm{H} 15^{\mathrm{x}}$ & 3.1438 \\
\hline $\mathrm{H} 14 \cdots \mathrm{Cl}^{\mathrm{v}}$ & 3.3665 \\
\hline $\mathrm{H} 14 \cdots \mathrm{Cl} 2^{\mathrm{xi}}$ & 3.1376 \\
\hline
\end{tabular}




\begin{tabular}{|c|c|c|c|}
\hline $\mathrm{O} 1 \cdots \mathrm{H} 11^{\mathrm{vi}}$ & 3.1669 & $\mathrm{H} 14 \cdots \mathrm{C}^{\mathrm{xi}}$ & 3.5789 \\
\hline $\mathrm{O} 2 \cdots \mathrm{H} 2 \mathrm{~A}^{\mathrm{ii}}$ & 2.5248 & $\mathrm{H} 14 \cdots \mathrm{C} 16^{\mathrm{xii}}$ & 3.3549 \\
\hline $\mathrm{O} 2 \cdots \mathrm{H} 2 \mathrm{~A}^{\mathrm{i}}$ & 2.9081 & $\mathrm{H} 14 \cdots \mathrm{C} 17^{\mathrm{xii}}$ & 3.2288 \\
\hline $\mathrm{O} 2 \cdots \mathrm{H} 5^{\mathrm{ii}}$ & 2.7607 & $\mathrm{H} 14 \cdots \cdot \mathrm{H} 4^{\mathrm{xi}}$ & 2.7925 \\
\hline $\mathrm{O} 2 \cdots \mathrm{H} 6^{\mathrm{iii}}$ & 2.5402 & $\mathrm{H} 14 \cdots \mathrm{H} 4^{\mathrm{v}}$ & 3.5715 \\
\hline $\mathrm{O} 2 \cdots \mathrm{H} 7 \mathrm{~A}^{\mathrm{iii}}$ & 3.3604 & $\mathrm{H} 14 \cdots \mathrm{H} 12^{\mathrm{xii}}$ & 3.0781 \\
\hline $\mathrm{O} 2 \cdots \mathrm{H} 9^{\mathrm{iii}}$ & 3.5379 & $\mathrm{H} 14 \cdots \mathrm{H} 13^{\mathrm{xii}}$ & 2.8458 \\
\hline $\mathrm{N} 1 \cdots \mathrm{H} 2 \mathrm{~A}^{\mathrm{ii}}$ & 3.1678 & $\mathrm{H} 15 \cdots \mathrm{Cl} 1^{\mathrm{v}}$ & 3.3698 \\
\hline $\mathrm{N} 1 \cdots \mathrm{H} 2 \mathrm{~A}^{\mathrm{i}}$ & 3.5686 & $\mathrm{H} 15 \cdots \mathrm{Cl} 2^{\mathrm{ii}}$ & 3.2700 \\
\hline $\mathrm{N} 1 \cdots \mathrm{H} 10^{\text {iv }}$ & 3.3222 & $\mathrm{H} 15 \cdots \mathrm{C} 5^{v}$ & 3.4109 \\
\hline $\mathrm{C} 1 \cdots \mathrm{H} 6^{\mathrm{v}}$ & 3.2859 & $\mathrm{H} 15 \cdots \mathrm{H} 13^{\mathrm{xii}}$ & 3.1438 \\
\hline $\mathrm{C} 1 \cdots \mathrm{H}^{\text {iv }}$ & 3.0434 & & \\
\hline $\mathrm{C} 1-\mathrm{O} 1-\mathrm{C} 8$ & $112.82(12)$ & $\mathrm{C} 19-\mathrm{C} 20-\mathrm{C} 21$ & $118.39(13)$ \\
\hline $\mathrm{C} 10-\mathrm{N} 1-\mathrm{C} 11$ & $121.31(12)$ & $\mathrm{C} 15-\mathrm{C} 21-\mathrm{C} 16$ & $121.33(15)$ \\
\hline $\mathrm{O} 1-\mathrm{C} 1-\mathrm{C} 2$ & $112.00(12)$ & $\mathrm{C} 15-\mathrm{C} 21-\mathrm{C} 20$ & $119.55(14)$ \\
\hline $\mathrm{C} 1-\mathrm{C} 2-\mathrm{C} 3$ & $117.41(13)$ & $\mathrm{C} 16-\mathrm{C} 21-\mathrm{C} 20$ & $119.12(13)$ \\
\hline $\mathrm{C} 1-\mathrm{C} 2-\mathrm{C} 10$ & $119.51(12)$ & $\mathrm{C} 10-\mathrm{N} 1-\mathrm{H} 6$ & 119.342 \\
\hline $\mathrm{C} 3-\mathrm{C} 2-\mathrm{C} 10$ & $122.82(14)$ & $\mathrm{C} 11-\mathrm{N} 1-\mathrm{H} 6$ & 119.346 \\
\hline $\mathrm{O} 2-\mathrm{C} 3-\mathrm{C} 2$ & $124.70(13)$ & $\mathrm{O} 1-\mathrm{C} 1-\mathrm{H} 1 \mathrm{~B}$ & 109.210 \\
\hline $\mathrm{O} 2-\mathrm{C} 3-\mathrm{C} 9$ & $120.73(12)$ & $\mathrm{O} 1-\mathrm{C} 1-\mathrm{H} 2 \mathrm{~A}$ & 109.215 \\
\hline $\mathrm{C} 2-\mathrm{C} 3-\mathrm{C} 9$ & $114.49(13)$ & $\mathrm{C} 2-\mathrm{C} 1-\mathrm{H} 1 \mathrm{~B}$ & 109.214 \\
\hline $\mathrm{C} 5-\mathrm{C} 4-\mathrm{C} 9$ & $119.64(14)$ & $\mathrm{C} 2-\mathrm{C} 1-\mathrm{H} 2 \mathrm{~A}$ & 109.212 \\
\hline $\mathrm{C} 11-\mathrm{C} 5-\mathrm{C} 4$ & $119.70(12)$ & $\mathrm{H} 1 \mathrm{~B}-\mathrm{C} 1-\mathrm{H} 2 \mathrm{~A}$ & 107.905 \\
\hline $\mathrm{C} 11-\mathrm{C} 5-\mathrm{C} 6$ & $118.85(11)$ & $\mathrm{C} 5-\mathrm{C} 4-\mathrm{H} 3$ & 120.177 \\
\hline $\mathrm{C} 4-\mathrm{C} 5-\mathrm{C} 6$ & $121.43(12)$ & $\mathrm{C} 9-\mathrm{C} 4-\mathrm{H} 3$ & 120.180 \\
\hline $\mathrm{C} 5-\mathrm{C} 6-\mathrm{C} 7$ & $118.60(13)$ & $\mathrm{C} 5-\mathrm{C} 6-\mathrm{H} 4$ & 120.699 \\
\hline $\mathrm{C} 12-\mathrm{C} 7-\mathrm{C} 6$ & $119.28(11)$ & $\mathrm{C} 7-\mathrm{C} 6-\mathrm{H} 4$ & 120.701 \\
\hline $\mathrm{Cl} 2-\mathrm{C} 7-\mathrm{C} 8$ & $119.20(10)$ & $\mathrm{N} 1-\mathrm{C} 10-\mathrm{H} 5$ & 116.499 \\
\hline $\mathrm{C} 6-\mathrm{C} 7-\mathrm{C} 8$ & $121.52(13)$ & $\mathrm{C} 2-\mathrm{C} 10-\mathrm{H} 5$ & 116.493 \\
\hline $\mathrm{O} 1-\mathrm{C} 8-\mathrm{C} 7$ & $118.23(13)$ & $\mathrm{N} 1-\mathrm{C} 11-\mathrm{H} 7 \mathrm{~A}$ & 108.592 \\
\hline $\mathrm{O} 1-\mathrm{C} 8-\mathrm{C} 9$ & $122.75(13)$ & $\mathrm{N} 1-\mathrm{C} 11-\mathrm{H} 8 \mathrm{~B}$ & 108.590 \\
\hline $\mathrm{C} 7-\mathrm{C} 8-\mathrm{C} 9$ & $118.88(12)$ & $\mathrm{C} 12-\mathrm{C} 11-\mathrm{H} 7 \mathrm{~A}$ & 108.590 \\
\hline $\mathrm{C} 3-\mathrm{C} 9-\mathrm{C} 4$ & $120.27(13)$ & $\mathrm{C} 12-\mathrm{C} 11-\mathrm{H} 8 \mathrm{~B}$ & 108.589 \\
\hline $\mathrm{C} 3-\mathrm{C} 9-\mathrm{C} 8$ & $119.48(12)$ & $\mathrm{H} 7 \mathrm{~A}-\mathrm{C} 11-\mathrm{H} 8 \mathrm{~B}$ & 107.556 \\
\hline $\mathrm{C} 4-\mathrm{C} 9-\mathrm{C} 8$ & $119.90(13)$ & $\mathrm{C} 12-\mathrm{C} 13-\mathrm{H} 9$ & 119.454 \\
\hline $\mathrm{N} 1-\mathrm{C} 10-\mathrm{C} 2$ & $127.01(13)$ & $\mathrm{C} 14-\mathrm{C} 13-\mathrm{H} 9$ & 119.453 \\
\hline $\mathrm{N} 1-\mathrm{C} 11-\mathrm{C} 12$ & $114.70(11)$ & $\mathrm{C} 13-\mathrm{C} 14-\mathrm{H} 10$ & 119.980 \\
\hline $\mathrm{C} 11-\mathrm{C} 12-\mathrm{C} 13$ & $121.42(13)$ & $\mathrm{C} 15-\mathrm{C} 14-\mathrm{H} 10$ & 119.986 \\
\hline $\mathrm{C} 11-\mathrm{C} 12-\mathrm{C} 20$ & $118.60(11)$ & $\mathrm{C} 14-\mathrm{C} 15-\mathrm{H} 11$ & 119.628 \\
\hline $\mathrm{C} 13-\mathrm{C} 12-\mathrm{C} 20$ & $119.98(14)$ & $\mathrm{C} 21-\mathrm{C} 15-\mathrm{H} 11$ & 119.617 \\
\hline $\mathrm{C} 12-\mathrm{C} 13-\mathrm{C} 14$ & $121.09(14)$ & $\mathrm{C} 17-\mathrm{C} 16-\mathrm{H} 12$ & 119.532 \\
\hline $\mathrm{C} 13-\mathrm{C} 14-\mathrm{C} 15$ & $120.03(13)$ & $\mathrm{C} 21-\mathrm{C} 16-\mathrm{H} 12$ & 119.524 \\
\hline $\mathrm{C} 14-\mathrm{C} 15-\mathrm{C} 21$ & $120.75(15)$ & $\mathrm{C} 16-\mathrm{C} 17-\mathrm{H} 13$ & 119.901 \\
\hline $\mathrm{C} 17-\mathrm{C} 16-\mathrm{C} 21$ & $120.94(15)$ & $\mathrm{C} 18-\mathrm{C} 17-\mathrm{H} 13$ & 119.892 \\
\hline $\mathrm{C} 16-\mathrm{C} 17-\mathrm{C} 18$ & $120.21(14)$ & $\mathrm{C} 17-\mathrm{C} 18-\mathrm{H} 14$ & 119.822 \\
\hline $\mathrm{C} 17-\mathrm{C} 18-\mathrm{C} 19$ & $120.35(13)$ & $\mathrm{C} 19-\mathrm{C} 18-\mathrm{H} 14$ & 119.826 \\
\hline $\mathrm{C} 18-\mathrm{C} 19-\mathrm{C} 20$ & $120.98(14)$ & $\mathrm{C} 18-\mathrm{C} 19-\mathrm{H} 15$ & 119.511 \\
\hline
\end{tabular}


$\mathrm{C} 12-\mathrm{C} 20-\mathrm{C} 19$

$\mathrm{C} 12-\mathrm{C} 20-\mathrm{C} 21$

$\mathrm{C} 1-\mathrm{O} 1-\mathrm{C} 8-\mathrm{C} 7$

$\mathrm{C} 1-\mathrm{O} 1-\mathrm{C} 8-\mathrm{C} 9$

$\mathrm{C} 8-\mathrm{O} 1-\mathrm{C} 1-\mathrm{C} 2$

$\mathrm{C} 8-\mathrm{O} 1-\mathrm{C} 1-\mathrm{H} 1 \mathrm{~B}$

$\mathrm{C} 8-\mathrm{O} 1-\mathrm{C} 1-\mathrm{H} 2 \mathrm{~A}$

$\mathrm{C} 10-\mathrm{N} 1-\mathrm{C} 11-\mathrm{C} 12$

$\mathrm{C} 10-\mathrm{N} 1-\mathrm{C} 11-\mathrm{H} 7 \mathrm{~A}$

$\mathrm{C} 10-\mathrm{N} 1-\mathrm{C} 11-\mathrm{H} 8 \mathrm{~B}$

$\mathrm{C} 11-\mathrm{N} 1-\mathrm{C} 10-\mathrm{C} 2$

$\mathrm{C} 11-\mathrm{N} 1-\mathrm{C} 10-\mathrm{H} 5$

$\mathrm{H} 6-\mathrm{N} 1-\mathrm{C} 10-\mathrm{C} 2$

H6-N1- $110-\mathrm{H} 5$

H6-N1- $111-\mathrm{C} 12$

$\mathrm{H} 6-\mathrm{N} 1-\mathrm{C} 11-\mathrm{H} 7 \mathrm{~A}$

$\mathrm{H} 6-\mathrm{N} 1-\mathrm{C} 11-\mathrm{H} 8 \mathrm{~B}$

$\mathrm{O} 1-\mathrm{C} 1-\mathrm{C} 2-\mathrm{C} 3$

$\mathrm{O} 1-\mathrm{C} 1-\mathrm{C} 2-\mathrm{C} 10$

$\mathrm{H} 1 \mathrm{~B}-\mathrm{C} 1-\mathrm{C} 2-\mathrm{C} 3$

$\mathrm{H} 1 \mathrm{~B}-\mathrm{C} 1-\mathrm{C} 2-\mathrm{C} 10$

$\mathrm{H} 2 \mathrm{~A}-\mathrm{C} 1-\mathrm{C} 2-\mathrm{C} 3$

$\mathrm{H} 2 \mathrm{~A}-\mathrm{C} 1-\mathrm{C} 2-\mathrm{C} 10$

$\mathrm{C} 1-\mathrm{C} 2-\mathrm{C} 3-\mathrm{O} 2$

$\mathrm{C} 1-\mathrm{C} 2-\mathrm{C} 3-\mathrm{C} 9$

$\mathrm{C} 1-\mathrm{C} 2-\mathrm{C} 10-\mathrm{N} 1$

$\mathrm{C} 1-\mathrm{C} 2-\mathrm{C} 10-\mathrm{H} 5$

$\mathrm{C} 3-\mathrm{C} 2-\mathrm{C} 10-\mathrm{N} 1$

$\mathrm{C} 3-\mathrm{C} 2-\mathrm{C} 10-\mathrm{H} 5$

$\mathrm{C} 10-\mathrm{C} 2-\mathrm{C} 3-\mathrm{O} 2$

$\mathrm{C} 10-\mathrm{C} 2-\mathrm{C} 3-\mathrm{C} 9$

$\mathrm{O} 2-\mathrm{C} 3-\mathrm{C} 9-\mathrm{C} 4$

$\mathrm{O} 2-\mathrm{C} 3-\mathrm{C} 9-\mathrm{C} 8$

$\mathrm{C} 2-\mathrm{C} 3-\mathrm{C} 9-\mathrm{C} 4$

$\mathrm{C} 2-\mathrm{C} 3-\mathrm{C} 9-\mathrm{C} 8$

$\mathrm{C} 5-\mathrm{C} 4-\mathrm{C} 9-\mathrm{C} 3$

$\mathrm{C} 5-\mathrm{C} 4-\mathrm{C} 9-\mathrm{C} 8$

$\mathrm{C} 9-\mathrm{C} 4-\mathrm{C} 5-\mathrm{Cl} 1$

$\mathrm{C} 9-\mathrm{C} 4-\mathrm{C} 5-\mathrm{C} 6$

$\mathrm{H} 3-\mathrm{C} 4-\mathrm{C} 5-\mathrm{Cl1}$

$\mathrm{H} 3-\mathrm{C} 4-\mathrm{C} 5-\mathrm{C} 6$

$\mathrm{H} 3-\mathrm{C} 4-\mathrm{C} 9-\mathrm{C} 3$

$\mathrm{H} 3-\mathrm{C} 4-\mathrm{C} 9-\mathrm{C} 8$

$\mathrm{C} 11-\mathrm{C} 5-\mathrm{C} 6-\mathrm{C} 7$

$\mathrm{Cl1}-\mathrm{C} 5-\mathrm{C} 6-\mathrm{H} 4$

$\mathrm{C} 4-\mathrm{C} 5-\mathrm{C} 6-\mathrm{C} 7$

$\mathrm{C} 4-\mathrm{C} 5-\mathrm{C} 6-\mathrm{H} 4$
$123.04(13)$

118.57 (12)

$155.30(11)$

$-29.15(17)$

52.17 (15)

$-68.9$

173.3

$-98.17(16)$

140.2

23.5

$-175.34(12)$

4.7

4.7

$-175.3$

81.8

$-39.8$

$-156.5$

-44.51 (16)

$141.16(11)$

76.6

$-97.7$

$-165.6$

20.1

$-171.00(12)$

$12.11(17)$

171.75 (12)

$-8.3$

$-2.3(3)$

177.7

$3.1(3)$

$-173.76(12)$

8.74 (19)

$-164.33(12)$

$-174.23(11)$

12.69 (17)

$-172.09(11)$

$1.0(2)$

$179.53(11)$

0.7 (2)

$-0.5$

$-179.3$

7.9

$-179.0$

$179.64(9)$

$-0.4$

$-1.6(2)$

178.4
$\mathrm{C} 20-\mathrm{C} 19-\mathrm{H} 15$

119.512

$\mathrm{O} 1-\mathrm{C} 8-\mathrm{C} 9-\mathrm{C} 4$

$\mathrm{C} 7-\mathrm{C} 8-\mathrm{C} 9-\mathrm{C} 3$

$\mathrm{C} 7-\mathrm{C} 8-\mathrm{C} 9-\mathrm{C} 4$

$\mathrm{N} 1-\mathrm{C} 11-\mathrm{C} 12-\mathrm{C} 13$

$\mathrm{N} 1-\mathrm{C} 11-\mathrm{C} 12-\mathrm{C} 20$

$\mathrm{H} 7 \mathrm{~A}-\mathrm{C} 11-\mathrm{C} 12-\mathrm{C} 13$

$\mathrm{H} 7 \mathrm{~A}-\mathrm{C} 11-\mathrm{C} 12-\mathrm{C} 20$

$\mathrm{H} 8 \mathrm{~B}-\mathrm{C} 11-\mathrm{C} 12-\mathrm{C} 13$

$\mathrm{H} 8 \mathrm{~B}-\mathrm{C} 11-\mathrm{C} 12-\mathrm{C} 20$

$\mathrm{C} 11-\mathrm{C} 12-\mathrm{C} 13-\mathrm{C} 14$

$\mathrm{C} 11-\mathrm{C} 12-\mathrm{C} 13-\mathrm{H} 9$

$\mathrm{C} 11-\mathrm{C} 12-\mathrm{C} 20-\mathrm{C} 19$

$\mathrm{C} 11-\mathrm{C} 12-\mathrm{C} 20-\mathrm{C} 21$

$\mathrm{C} 13-\mathrm{C} 12-\mathrm{C} 20-\mathrm{C} 19$

$\mathrm{C} 13-\mathrm{C} 12-\mathrm{C} 20-\mathrm{C} 21$

$\mathrm{C} 20-\mathrm{C} 12-\mathrm{C} 13-\mathrm{C} 14$

$\mathrm{C} 20-\mathrm{C} 12-\mathrm{C} 13-\mathrm{H} 9$

$\mathrm{C} 12-\mathrm{C} 13-\mathrm{C} 14-\mathrm{C} 15$

$\mathrm{C} 12-\mathrm{C} 13-\mathrm{C} 14-\mathrm{H} 10$

H9-C13-C14-C15

$\mathrm{H} 9-\mathrm{C} 13-\mathrm{C} 14-\mathrm{H} 10$

$\mathrm{C} 13-\mathrm{C} 14-\mathrm{C} 15-\mathrm{C} 21$

$\mathrm{C} 13-\mathrm{C} 14-\mathrm{C} 15-\mathrm{H} 11$

$\mathrm{H} 10-\mathrm{C} 14-\mathrm{C} 15-\mathrm{C} 21$

$\mathrm{H} 10-\mathrm{C} 14-\mathrm{C} 15-\mathrm{H} 11$

$\mathrm{C} 14-\mathrm{C} 15-\mathrm{C} 21-\mathrm{C} 16$

$\mathrm{C} 14-\mathrm{C} 15-\mathrm{C} 21-\mathrm{C} 20$

$\mathrm{H} 11-\mathrm{C} 15-\mathrm{C} 21-\mathrm{C} 16$

$\mathrm{H} 11-\mathrm{C} 15-\mathrm{C} 21-\mathrm{C} 20$

$\mathrm{C} 17-\mathrm{C} 16-\mathrm{C} 21-\mathrm{C} 15$

$\mathrm{C} 17-\mathrm{C} 16-\mathrm{C} 21-\mathrm{C} 20$

$\mathrm{C} 21-\mathrm{C} 16-\mathrm{C} 17-\mathrm{C} 18$

$\mathrm{C} 21-\mathrm{C} 16-\mathrm{C} 17-\mathrm{H} 13$

$\mathrm{H} 12-\mathrm{C} 16-\mathrm{C} 17-\mathrm{C} 18$

$\mathrm{H} 12-\mathrm{C} 16-\mathrm{C} 17-\mathrm{H} 13$

$\mathrm{H} 12-\mathrm{C} 16-\mathrm{C} 21-\mathrm{C} 15$

$\mathrm{H} 12-\mathrm{C} 16-\mathrm{C} 21-\mathrm{C} 20$

$\mathrm{C} 16-\mathrm{C} 17-\mathrm{C} 18-\mathrm{C} 19$

C16-C17-C18-H14

H13-C17-C18-C19

$\mathrm{H} 13-\mathrm{C} 17-\mathrm{C} 18-\mathrm{H} 14$

$\mathrm{C} 17-\mathrm{C} 18-\mathrm{C} 19-\mathrm{C} 20$

C17-C18-C19-H15

$\mathrm{H} 14-\mathrm{C} 18-\mathrm{C} 19-\mathrm{C} 20$

H14-C18-C19-H15
$-177.29(11)$

$171.33(11)$

$-1.77(19)$

$-6.86(19)$

174.05 (11)

114.8

$-64.3$

$-128.5$

52.4

$-178.41(12)$

1.6

-2.90 (19)

177.44 (11)

178.00 (12)

$-1.66(19)$

0.7 (2)

$-179.3$

$0.5(3)$

$-179.5$

$-179.5$

0.5

$-0.6(3)$

179.4

179.4

$-0.6$

$-179.85(13)$

-0.4 (3)

0.1

179.6

178.49 (13)

-0.9 (3)

-0.1 (3)

179.9

179.9

$-0.1$

$-1.5$

179.1

0.7 (3)

$-179.3$

$-179.3$

0.7

$-0.3(3)$

179.7

179.7

$-0.3$ 


\section{supporting information}

$\begin{array}{llll}\mathrm{C} 5-\mathrm{C} 6-\mathrm{C} 7-\mathrm{C} 2 & 179.84(11) & \mathrm{C} 18-\mathrm{C} 19-\mathrm{C} 20-\mathrm{C} 12 & 179.65(12) \\ \mathrm{C} 5-\mathrm{C} 6-\mathrm{C} 7-\mathrm{C} 8 & 0.70(19) & \mathrm{C} 18-\mathrm{C} 19-\mathrm{C} 20-\mathrm{C} 21 & -0.7(2) \\ \mathrm{H} 4-\mathrm{C} 6-\mathrm{C} 7-\mathrm{C} 2 & -0.2 & \mathrm{H} 15-\mathrm{C} 19-\mathrm{C} 20-\mathrm{C} 12 & -0.3 \\ \mathrm{H} 4-\mathrm{C} 6-\mathrm{C} 7-\mathrm{C} 8 & -179.3 & \mathrm{H} 15-\mathrm{C} 19-\mathrm{C} 20-\mathrm{C} 21 & 179.3 \\ \mathrm{C} 2-\mathrm{C} 7-\mathrm{C} 8-\mathrm{O} 1 & -2.47(17) & \mathrm{C} 12-\mathrm{C} 20-\mathrm{C} 21-\mathrm{C} 15 & 1.54(19) \\ \mathrm{C} 12-\mathrm{C} 7-\mathrm{C} 8-\mathrm{C} 9 & -178.20(8) & \mathrm{C} 12-\mathrm{C} 20-\mathrm{C} 21-\mathrm{C} 16 & -179.02(11) \\ \mathrm{C} 6-\mathrm{C} 7-\mathrm{C} 8-\mathrm{O} 1 & 176.67(12) & \mathrm{C} 19-\mathrm{C} 20-\mathrm{C} 21-\mathrm{C} 15 & -178.13(12) \\ \mathrm{C} 6-\mathrm{C} 7-\mathrm{C} 8-\mathrm{C} 9 & 0.94(19) & \mathrm{C} 19-\mathrm{C} 20-\mathrm{C} 21-\mathrm{C} 16 & 1.30(19) \\ \mathrm{O} 1-\mathrm{C} 8-\mathrm{C} 9-\mathrm{C} 3 & -4.19(19) & & \end{array}$

Symmetry codes: (i) $x,-y+3 / 2, z-1 / 2$; (ii) $-x+1, y-1 / 2,-z+3 / 2$; (iii) $-x+1,-y+1,-z+1$; (iv) $x,-y+3 / 2, z+1 / 2$; (v) $-x+1, y+1 / 2,-z+3 / 2$; (vi) $-x+1,-y+2$, $-z+1$; (vii) $-x,-y+1,-z+1$; (viii) $x-1, y, z$; (ix) $-x+2,-y+2,-z+1$; (x) $-x+2, y+1 / 2,-z+3 / 2$; (xi) $x+1, y, z$; (xii) $-x+2, y-1 / 2,-z+3 / 2$.

Hydrogen-bond geometry $\left(\AA,{ }^{\circ}\right)$

$\mathrm{Cg} 2$ is the centroid of the $\mathrm{C} 4-\mathrm{C} 9$ ring.

\begin{tabular}{lllll}
\hline$D-\mathrm{H} \cdots A$ & $D-\mathrm{H}$ & $\mathrm{H} \cdots A$ & $D \cdots A$ & $D-\mathrm{H} \cdots A$ \\
\hline $\mathrm{N} 1-\mathrm{H} 6 \cdots \mathrm{O} 2$ & 0.88 & 2.18 & $2.794(2)$ & 126 \\
$\mathrm{~N} 1-\mathrm{H} 6 \cdots \mathrm{O} 2^{\mathrm{iii}}$ & 0.88 & 2.54 & $3.306(3)$ & 146 \\
$\mathrm{C} 1-\mathrm{H} 2 A \cdots \mathrm{O} 2^{\mathrm{v}}$ & 0.99 & 2.52 & $3.472(3)$ & 160 \\
$\mathrm{C} 15-\mathrm{H} 11 \cdots \mathrm{Cg} 2^{\mathrm{vi}}$ & 0.95 & 2.77 & $3.682(3)$ & 160
\end{tabular}

Symmetry codes: (iii) $-x+1,-y+1,-z+1$; (v) $-x+1, y+1 / 2,-z+3 / 2$; (vi) $-x+1,-y+2,-z+1$. 